\title{
Linear instability driven by an electric field in two-layer channel flow of Newtonian and Herschel-Bulkley fluids
}

\author{
K. Gautam ${ }^{\text {a }}$, P.A.L. Narayana ${ }^{\text {a }}$, Kirti Chandra Sahu ${ }^{\text {b,* }}$ \\ a Department of Mathematics, Indian Institute of Technology Hyderabad, Sangareddy, 502285, Telangana, India \\ ${ }^{\mathrm{b}}$ Department of Chemical Engineering, Indian Institute of Technology Hyderabad, Sangareddy, 502 285, Telangana, India
}

\section{A R T I C L E I N F O}

\section{Keywords:}

Linear instability

Herschel-Bulkley fluid

Electric field

Interface

Channel flow

\begin{abstract}
A B S T R A C T
We investigate the linear stability characteristics of a pressure-driven two-layer channel flow of immiscible Newtonian and Herschel-Bulkley fluids subjected to an applied electric field normal to the flow. The linear stability equations are derived and solved using an accurate spectral Chebyshev collocation method. It is found that the electric field can stabilise or destabilise the flow depending on the electrical properties of the fluids. We also observe that increasing the electric permittivity ratio and decreasing the electrical conductivity ratio, while keeping the rest of the parameters constant, enhances the growth rate of the disturbances. The "Reynolds stress" of the Newtonian layer and the work done by the velocity and stress disturbances tangential to the interface are found to be the mechanism of the instability observed due to the applied electric field. A parametric study is also conducted by varying the thickness of the bottom layer, Bingham number and flow index of the Herschel-Bulkley fluid. Increasing Bingham number is found to be stabilising or destabilising depending on the thickness of the non-Newtonian layer and the maximum disturbance growth occurs at an optimum value of non-Newtonian layer thicknesses. Increasing the shear-thinning and shear-thickening nature is shown to destabilise the flow. Our study is relevant in many microfluidic and electronic cooling applications.
\end{abstract}

\section{Introduction}

Interfacial instability in the flows involving two superposed fluid layers has been a topic of interest for decades due to its relevance in many engineering applications [1,2]. Thus, a large number of earlier studies considered the linear stability characteristics of Newtonian [39] and non-Newtonian Herschel-Bulkley fluids [10-16] in two-layer channel/pipe flows. The main findings of these studies are (i) the interfacial instability appears due to viscosity stratification even at a very small Reynolds number, (ii) the work done by the velocity and stress disturbances in the direction tangential to the interface is the mechanism of this instability for both Newtonian and non-Newtonian fluids, (iii) for Bingham fluids, the yield stress either stabilises and destabilises interfacial instability depending on the presence and absence of an unyielded region in the flow. A review of the literature on this subject can be found in Govindarajan and Sahu [17]. All of these studies are for configurations in the absence of electric field.

The electrical field can be used in controlling the instability that was addressed in the pioneering work of Taylor and McEwan [18] and Melcher and Smith [19], and subsequently extended by many researchers for Newtonian fluids (see, for instance, Ref. [20] and references therein). The application of electric field on multiphase flows includes the generation of droplets in microchannels [21], enhancing the mixing in micro-electro-mechanical systems (MEMS) [22-24], to name a few. Two types of configurations were considered to study the effect of the electrical field on the instability in superimposed fluid layers, namely, bulk-coupled configuration [25] where the electrical fluid properties vary in a thin diffusion layer and surface-coupled configuration [26] where the electrical fluid properties are subjected to a sudden jump across the interface. The present study considers a surface-coupled configuration. In the latter configuration, assuming rapid relaxation time scale, Thaokar and Kumaran [27] used linear and weakly nonlinear analyses to investigate the stability of the interface between two dielectric fluids confined between two parallel plates subject to an electrical field in the Stokes flow limit. Ozen et al. [28] conducted a linear stability analysis to study the interfacial instability of two Newtonian fluids in a plane Poiseuille flow subject to a normal electrical field in the limit of fast charge relaxation time scale. They found that the applied electric field can suppress or amplify the interfacial instability for different parameter values.

Several researchers have also studied the influence of the electric field on the stability of non-Newtonian fluids of different rheologies, due to its relevance in many practical applications (e.g. see Ref. [29]).

\footnotetext{
* Corresponding author.

E-mail address: ksahu@che.iith.ac.in (K.C. Sahu).
} 
Moreover, there has been a growing research interest in this topic [3032]. In a two-layer Couette flow of two dielectric Oldroyd-B fluids, Eldabe [33] conducted a long-wave stability analysis to investigate the effect of electric field applied normal to the flow. This was extended to power-law fluids in Ref. [34]. In these studies, it was reported that the electric field can have a stabilising or destabilising influence. Ersoy and Uguz [35] studied the linear stability of the interface between a Newtonian fluid and non-Newtonian fluid characterised by the upperconvected Maxwell model subjected to a normal electric field. They also found that increasing the applied voltage could stabilise or destabilise the flow depending on the electrical properties of the liquids. Nurocak and Uguz [36] investigated the effect of an electric field applied along or normal to an interface between an upper-convected Maxwell fluid and a passive fluid. Recently, the linear instability characteristic of a flat interface between Newtonian and power-law fluids has been investigated by Kaykanat and Uguz [37]. They observed that the kinematic boundary condition at the interface is the main source of instability when the charge relaxation time is quick.

In this study, we examine the linear stability characteristics of Newtonian and Herschel-Bulkley fluids in a two-layer configuration that is subjected to an electric field normal to the flow. In order to isolate the effect of the electric field, the fluids are assumed to have the same density. As the fluids are immiscible, the fluid and electrical properties undergo sudden jump across the interface. The linear stability equations are derived and solved using a spectral Chebyshev collocation method. We found that the electric field has a destabilising influence on the flow for the parameters considered in the present study. The "Reynolds stress" of the Newtonian layer and the work done by the velocity and stress disturbances tangential to the interface are found to be the mechanism of the instability observed. The enhancement of the instability due to the applied electric field is also examined by plotting the disturbance eigenfunctions profiles. We have also conducted a parametric study by varying the electric permittivity and conductivity ratios, the thickness of the bottom layer, the Bingham number and the flow index of the Herschel-Bulkley fluid layer. It is found that increasing Bingham number can be stabilising or destabilising depending on the thickness of the non-Newtonian layer. Increasing the shear-thinning and shear-thickening nature of the non-Newtonian layer is also shown to destabilise the flow. Moreover, increasing the flow index increases the range of unstable wavenumbers in the presence and absence of electrical field.

The rest of the article is organised as follows. The problem is formulated in Section 2, wherein the basic state, the linear stability equations are provided and the associated dimensionless numbers are presented. In Section 3, the linear stability results are discussed and an energy budget analysis is also conducted to understand the mechanism of the instability observed under the application of an electric field. The concluding remarks are given in Section 4.

\section{Formulation}

We study the linear instability in a two-layer pressure-driven channel flow of a Newtonian fluid (designated by fluid 2) and a nonNewtonian fluid (designated by fluid 1) under the influence of an electric field of electric potential $\phi_{0}$ applied orthogonally to the flow as shown in the schematic diagram (Fig. 1). It is assumed that the fluids are incompressible and immiscible and have the same density $(\rho)$. A rectangular coordinate system $(x, y)$ is used to modelled the system, where $x$ and $y$ denote the streamwise and wall-normal directions, respectively. The lower and upper channel walls are located at $y=0$ and $y=H$, respectively. The sharp interface that separates the fluids is at $y=h^{0}$. The dynamic viscosity, electrical conductivity and permittivity of fluid ' 1 ' and fluid ' 2 ' are $\left(\mu_{1}, \sigma_{1}, \epsilon_{1}\right)$ and $\left(\mu_{2}, \sigma_{2}, \epsilon_{2}\right)$, respectively. The interfacial tension is denoted by $\gamma$.

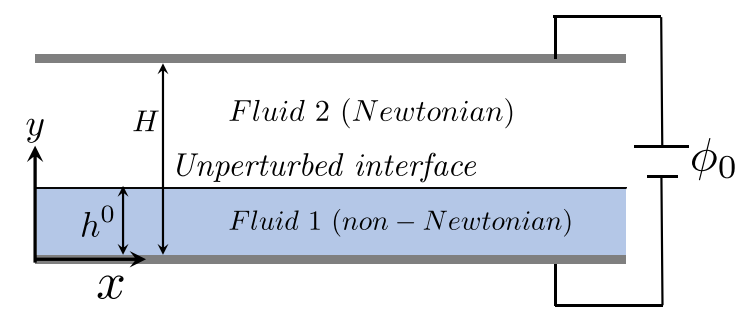

Fig. 1. The schematic diagram of the flow configuration considered. Fluid 1 is a non-Newtonian fluid characterised by a Herschel-Bulkley model, whereas Fluid 2 is a Newtonian fluid. The height of the channel is $H$ and the fluids are separated by a sharp interface at $y=h^{0}$. The wall at $y=0$ is grounded and an electric field of potential $\phi_{0}$ is applied between the walls in $y$ direction.

\subsection{Electric field}

Under the action of the applied electric field, the fluids experience electrostatic force/Coulomb force, which in turn effect the interfacial stresses (normal/tangential) at the interface separating the immiscible fluids. In the absence of magnetic field, the electric field can be assumed to be irrotational. Thus, the Gauss law of the volumetric surface charge density $(q)$ is given by

$\nabla \cdot\left(\epsilon_{i} \nabla \phi_{0}\right)=-q \quad(i=1,2)$.

The surface charge density around a fluid particle decays with a time scale, $t_{e}=\epsilon_{i} / \sigma_{i}$ (known as electric relaxation time). The viscous time scale is given by $t_{v}=\rho_{i} H^{2} / \mu_{i}$. In case of a conducting fluid with $t_{e} \ll t_{v}$, the charge accumulates at the interface almost instantaneously, i.e the charge conservation in the bulk fluid can reach to a steady state much faster than the fluid motion. When both fluids have low electrical conductivities, $t_{e} \gg t_{v}$, then the medium is known as perfect dielectric; i.e. in this case, $q=0$.

\subsection{Governing equations and nondimensionalisation}

The viscosity of the non-Newtonian fluid $\left(\mu_{1}\right)$ is characterised by the Herschel-Bulkley model, which is given by

$\mu_{1}=k \pi^{n-1}+\tau_{0} \pi^{-1}$,

where $k$ and $n$ denote the consistency and flow index, respectively; $\tau_{0}$ is the yield shear stress; $\pi \equiv\left(2 E_{i j} E_{i j}\right)^{1 / 2}$ represents the second invariant of the rate of strain tensor, $E_{i j}=\frac{1}{2}\left(\partial u_{i} / \partial x_{j}+\partial u_{j} / \partial x_{i}\right)$.

The flow dynamics is governed by the continuity, Navier-Stokes and Gauss's law of electric potential for each layer. The solution of these equations is sought subject to the conditions of no-slip and no-penetration on the walls, continuity of the normal and tangential components of the velocity and stress, and the kinematic condition at the interface. The following scaling is used to render the governing equations and boundary conditions non-dimensional.

$\left(x, y, h^{0}\right)=H\left(\tilde{x}, \tilde{y}, \widetilde{h}^{0}\right), t=H / V \widetilde{t}, \mathbf{u}=V \tilde{\mathbf{u}},\left(\phi_{1}, \phi_{2}\right)=\phi_{0}\left(\widetilde{\phi_{1}}, \widetilde{\phi_{2}}\right)$,

$p_{i}=\mu_{2} V \widetilde{P}_{i} / H,\left(\mu_{1}, \mu_{2}\right)=\mu_{2}\left(\widetilde{\mu_{1}}, \widetilde{\mu_{2}}\right),\left(\sigma_{1}, \sigma_{2}\right)=\sigma_{2}\left(\widetilde{\sigma_{1}}, \widetilde{\sigma_{2}}\right)$,

$\left(\epsilon_{1}, \epsilon_{2}\right)=\epsilon_{2}\left(\widetilde{\epsilon_{1}}, \widetilde{\epsilon_{2}}\right), q=q_{s} \widetilde{q}$,

where the superscript tilde designates the dimensionless quantity, and $\mathbf{u}(u, v)$ represents the velocity field, where $u$ and $v$ are the components of the velocity in the $x$ and $y$ directions, respectively. $V(\equiv Q / H)$, $q_{S}\left(\equiv \varepsilon_{2} \phi_{0} / H\right)$ and the fluid properties of the Newtonian fluid are used as the scales, wherein $Q$ denotes the total flow rate per unit width in the spanwise direction and $H$ is the height of the channel. After suppressing tilde notations, the dimensionless governing equations are given by

$u_{i, x}+v_{i, y}=0$,

$\operatorname{Re}\left(u_{i, t}+u_{i} u_{i, x}+v_{i} u_{i, y}\right)=-P_{i, x}+2\left(\mu_{i} u_{i, x}\right)_{x}+\left(\mu_{i}\left(u_{i, y}+v_{i, x}\right)\right)_{y}$, 
$\operatorname{Re}\left(v_{i, t}+u_{i} v_{i, x}+v_{i} v_{i, y}\right)=-P_{i, y}+\left(\mu_{i}\left(v_{i, x}+u_{i, y}\right)\right)_{x}+2\left(\mu_{i} v_{i, y}\right)_{y}$,

$\phi_{i, x x}+\phi_{i, y y}=0$,

where subscripts $t, x$ and $y$ represent the partial derivatives with respect to $t, x$ and $y$, respectively. The dimensionless viscosity of the non-Newtonian fluid $\left(\mu_{1}\right)$ is given by

$\mu_{1}=m \Pi^{n-1}+B n \Pi^{-1}$.

Here $R e \equiv \rho V H / \mu_{2}$ is the Reynolds number, $B n \equiv \tau_{0} H / \mu_{2} V$ is a Bingham number and $m \equiv \mu_{2}^{-1} k(V / H)^{n-1}$ is the Newtonian viscosity ratio.

The dimensionless boundary conditions at the walls are given by

$u_{1}=0, v_{1}=0, \phi_{1}=0$ at $y=0$,

$u_{2}=0, v_{2}=0, \phi_{2}=1$ at $y=1$.

The interfacial boundary conditions at the interface are given by:

(i) The continuity of the velocity components at the interface

$u_{1}=u_{2}, v_{1}=v_{2}$.

(ii) The continuity of the tangential components of the electric field across the interface

$\phi_{1, x}+h_{x} \phi_{1, y}=\phi_{2, x}+h_{x} \phi_{2, y}$.

(iii) Two additional interfacial boundary conditions for the surface charge density, $q$ are required. The first boundary condition comes from Gauss's law, i.e., the normal component of the electric field satisfies

$h_{x} \phi_{2, x}-\phi_{2, y}-\varepsilon_{r}\left(h_{x} \phi_{1, x}-\phi_{1, y}\right)=q\left(1+h_{x}^{2}\right)^{1 / 2}$.

(iv) The second boundary condition deals with accumulation of the charge particles at the interface due to the ionic conduction from the bulk fluid (leaky-dielectric model), which is given by

$$
\begin{aligned}
q_{t}+ & u_{1} q_{x}-\frac{1}{\left(1+h_{x}^{2}\right)}\left[h_{x}^{2} q_{x} u_{1}-h_{x} q_{x} v_{1}\right. \\
& \left.\quad+q\left\{h_{x}^{2} u_{1, x}-h_{x}\left(u_{1, y}+v_{1, x}\right)+v_{1, x}\right\}\right] \\
= & \frac{\Lambda}{\left(1+h_{x}^{2}\right)^{1 / 2}}\left[-h_{x} \phi_{2, x}+\phi_{2, y}-\sigma_{r}\left(-h_{x} \phi_{1, x}+\phi_{1, y}\right)\right] .
\end{aligned}
$$

(v) The normal and shear stresses balance equations are given by

$$
\begin{aligned}
& P_{1}-P_{2}+\frac{2}{\left(1+h_{x}^{2}\right)}\left[v_{2, y}+h_{x}^{2} u_{2, x}-h_{x}\left(u_{2, y}+v_{2, x}\right)\right] \\
& -\frac{2 \mu_{1}}{\left(1+h_{x}^{2}\right)}\left[v_{1, y}+h_{x}^{2} u_{1, x}-h_{x}\left(u_{1, y}+v_{1, x}\right)\right] \\
& +\frac{E_{0}}{2}\left[\left(h_{x}^{2}-1\right)\left\{\phi_{2, x}^{2}-\phi_{2, y}^{2}-\varepsilon_{r}\left(\phi_{1, x}^{2}-\phi_{1, y}^{2}\right)\right\}\right. \\
& \left.-4 h_{x}\left\{\phi_{2, x} \phi_{2, y}-\varepsilon_{r} \phi_{1, x} \phi_{1, y}\right\}\right] \\
& =-\frac{\Gamma}{\left(1+h_{x}^{2}\right)^{3 / 2}} h_{x x},
\end{aligned}
$$

and

$\left(1-h_{x}^{2}\right)\left(u_{2, y}+v_{2, x}\right)+2 h_{x}\left(v_{2, y}-u_{2, x}\right)-$

$\mu_{1}\left[\left(1-h_{x}^{2}\right)\left(u_{1, y}+v_{1, x}\right)+2 h_{x}\left(v_{1, y}-u_{1, x}\right)\right]=$

$-E_{0}\left[\left(h_{x} \phi_{2, x}-\phi_{2, y}\right)\left(\phi_{2, x}+h_{x} \phi_{2, y}\right)\right.$

$\left.-\varepsilon_{r}\left(h_{x} \phi_{1, x}-\phi_{1, y}\right)\left(\phi_{1, x}+h_{x} \phi_{1, y}\right)\right]$,

respectively. All the boundary conditions discussed so far are the dynamic boundary conditions.

(vi) The kinematic condition is given by

$h_{t}+u_{1} h_{x}=v_{1}$

where $\varepsilon_{r}\left(\equiv \varepsilon_{1} / \varepsilon_{2}\right)$ is the electrical permittivity ratio, $\sigma_{r}\left(\equiv \sigma_{1} / \sigma_{2}\right)$ is the electrical conductivity ratio, $\Lambda$ ( $\left.\equiv H \sigma_{2} / V \epsilon_{2}\right)$ is the fluid to electric
Table 1

The critical value of the Bingham number for different values of the flow index $(n)$. The rest of the parameters are $m=10$ and $h^{0}=0.5$.

\begin{tabular}{ll}
\hline Flow index $(n)$ & Critical Bingham number $\left(B n_{c r}\right)$ \\
\hline 0.7 & 11.0 \\
1.0 & 13.0 \\
1.2 & 13.4 \\
1.5 & 14.1 \\
\hline
\end{tabular}

time-scales and $E_{0}\left(\equiv \varepsilon_{2} \phi_{0}^{2} / \mu_{2} V H\right)$ is the electric Weber number. We restrict the analysis to sufficiently low values of $B n$ so that there are no unyielded regions in the flow domain. Note that in the above equations, by setting the electric field to zero, we can recover the governing equations and the boundary conditions reported in Refs. [14,15,38].

\subsection{Basic state}

The basic state velocity field is assumed to be steady and unidirectional driven by a linear pressure distribution in $x\left(P_{1}=P_{2} \equiv P\right)$. Thus, the basic state streamwise velocity profiles in both the layers can be obtained by integrating Eq. (5) under these assumptions. The streamwise velocity profiles in both the layers are given

$U_{1}=\frac{n}{n+1} \frac{P_{x}^{-1}}{m^{1 / n}}\left(P_{x} y+c_{3}-B n\right)^{(n+1) / n}+c_{4}$,

$U_{2}=P_{x} \frac{y^{2}}{2}+c_{1} y+c_{2}$.

The constant pressure gradient $\left(P_{x}\right)$ and the integration constants $\left(c_{1}\right.$, $c_{2}, c_{3}$, and $c_{4}$ ) are obtained by solving the following simultaneous equations resulting from the no-slip boundary conditions at the upper and lower walls, and demanding continuity of velocity at the interface separating the fluids $\left(y=h^{0}\right)$.

$\frac{n P_{x}^{-1}}{(n+1) m^{1 / n}}\left[\left(P_{x} h^{0}+c_{3}-B n\right)^{(n+1) / n}-\right.$

$\left.\left(c_{3}-B n\right)^{(n+1) / n}\right]-\frac{1}{2} P_{x}\left(h^{0^{2}}-1\right)-c_{1}\left(h^{0}-1\right)=0$,

$c_{3}=c_{1}, \quad c_{2}=-\frac{P_{x}}{2}-c_{1}, \quad c_{4}=-\frac{n P_{x}^{-1}}{(n+1) m^{1 / n}}\left(c_{1}-B n\right)^{(n+1) / n}$.

The condition for the constant volumetric flow rate is given by

$\int_{0}^{h^{0}} U_{1} d y+\int_{h^{0}}^{1} U_{2} d y=1$

$\phi_{1}^{0}=\frac{y}{\left(\sigma_{r}+h^{0}\left(1-\sigma_{r}\right)\right)}$,

The base state of the electric potential in each layer is given by

$\phi_{2}^{0}=\frac{\sigma_{r}}{\left(\sigma_{r}+h^{0}\left(1-\sigma_{r}\right)\right)} y+\frac{h^{0}\left(1-\sigma_{r}\right)}{\left(\sigma_{r}+h^{0}\left(1-\sigma_{r}\right)\right)}$,

and the constant charge density at the interface at $y=h^{0}$ obtained using the Gauss's law, which is given by

$q^{0}=\frac{\left(\varepsilon_{r}-\sigma_{r}\right)}{\left(\sigma_{r}+h^{0}\left(1-\sigma_{r}\right)\right)}$.

The basic state streamwise velocity profile, $U$ (i.e. $U_{1}$ and $U_{2}$ for the non-Newtonian and Newtonian layers, which are given by Eqs. (18) and (19), respectively) is a function of the Bingham number $(B n)$, flow index $(n)$, Newtonian viscosity ratio $(m)$ and the location of the interface $\left(h^{0}\right)$. In Figs. 2(a) and (b), the streamwise velocity profiles are plotted for different values of $B n$ for $h^{0}=0.3$ and $h^{0}=0.5$, respectively. The rest of the parameters are $n=1$ and $m=10$. It can be seen that increasing $B n$ decreases the gradient of the velocity profile of the non-Newtonian fluid layer and reaches to zero near the interface (unyielded region) for a critical value of $B n\left(B n_{c r}\right)$. The values of $B n_{c r}$ for different values of $n$ are given in Table 1 . 
(a)

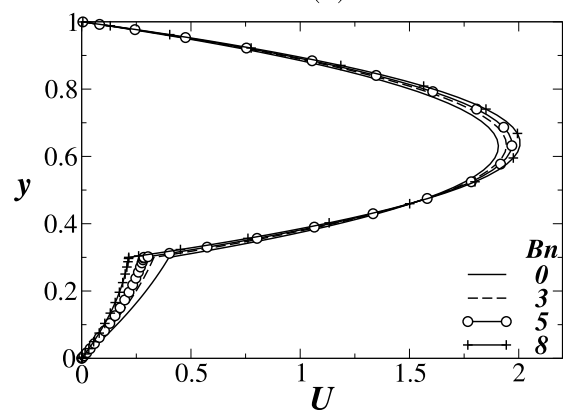

(b)

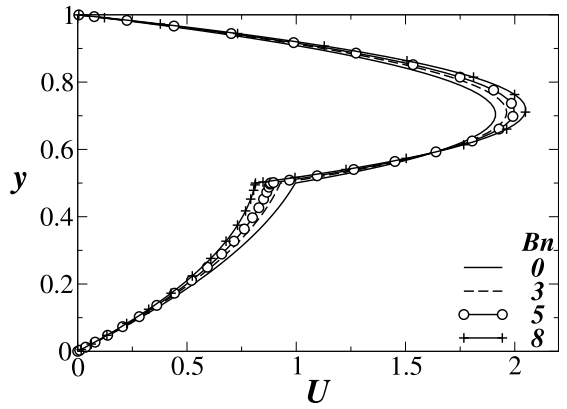

Fig. 2. The basic state streamwise velocity profile for different values of the Bingham number for $n=1$. (a) $h^{0}=0.5$ and (b) $h^{0}=0.3$.

\subsection{Linear stability analysis}

In order to study the linear stability characteristics of the basic state to infinitesimally small disturbances, the flow variables are represented as the sum of the basic state and two-dimensional disturbances as

$\left(u_{i}, v_{i}, P_{i}, \phi_{i}\right)(x, y, t)=\left[U_{i}(y), 0, P, \phi_{i}^{0}(y)\right]+\left(\hat{u}_{i}, \hat{v}_{i}, \hat{p}_{i}, \hat{\phi}_{i}\right)(x, y, t) \quad(i=1,2)$.

Similarly $\mu_{1}, h$, and $q$ can be perturbed as

$\mu_{1}(\pi)=\mu_{1}^{0}+\left.\frac{\partial \mu_{1}}{\partial \pi}\right|^{0}(\pi-\Pi) \equiv \mu_{1}^{0}+S \hat{\pi}$,

$h(x, t)=h^{0}+\hat{h}(x, t), \quad q(x, t)=q^{0}+\hat{q}(x, t)$,

where $S=(n-1) m \Pi^{n-2}-B n \Pi^{-2}$ and the superscript 0 designates the basic state quantities. Here, $\pi$ and $\hat{\pi}$ are the second invariant of the rate of strain tensor associated with the resulting flow after introducing the disturbance (i.e. basic flow + disturbance) and only disturbance, respectively. Substitution of Eqs. (26) and (27) into the governing equations, subtraction of the basic state equations, subsequent linearisation and elimination of the pressure perturbations yield the equations that govern the linear stability characteristics of the flow. These flow variables are then re-expressed in terms of the stream function as $\left(u_{i}, v_{i}\right)=\left(\partial \Psi_{i} / \partial y,-\partial \Psi_{i} / \partial x\right),(i=1,2)$ and decomposed using normal mode analysis as

$\left(\hat{u}_{i}, \hat{v}_{i}, \hat{p}_{i}, \hat{\pi}, \hat{\phi}_{i}\right)(x, y, t)=\left(\psi_{i}^{\prime},-i \alpha \psi_{i}, p_{i}, \pi_{i}, \phi_{i}\right)(y) e^{i(\alpha x-\omega t)}$,

$\hat{h}=\tilde{h} e^{i(\alpha x-\omega t)}, \quad \hat{q}=\tilde{q} e^{i(\alpha x-\omega t)}$,

where $\psi_{i}, \tilde{h}$ and $\tilde{q}$ denote the amplitude of the stream function, interfacial perturbation and surface charge density perturbation, respectively. The prime represents differentiation with respect to $y$. Here, $\alpha$ is a streamwise (real) wavenumber and $\omega(=\alpha c)$ is a complex frequency, wherein $c$ is a complex phase speed of the disturbance, such that $\omega_{i}>0$ or $c_{i}>0$ indicates the presence of a linear instability. The linear stability equations are given by

$$
\begin{aligned}
& i \alpha \operatorname{Re}\left[\left(\psi_{1}^{\prime \prime}-\alpha^{2} \psi_{1}\right)\left(U_{1}-c\right)-\psi_{1} U_{1}^{\prime \prime}\right]=\mu_{1}^{0}\left(\psi_{1}^{\prime \prime \prime \prime}-2 \alpha^{2} \psi_{1}^{\prime \prime}+\alpha^{4} \psi_{1}\right)+ \\
& 2 \mu_{1}^{0}\left(\psi_{1}^{\prime \prime \prime}-\alpha^{2} \psi_{1}^{\prime}\right)+\mu_{1}^{0 \prime \prime}\left(\psi_{1}^{\prime \prime}+\alpha^{2} \psi_{1}\right)+2 U_{1}^{\prime \prime}\left(S \pi^{\prime}+S^{\prime} \pi\right)+ \\
& \quad U_{1}^{\prime}\left(S \pi^{\prime \prime}+2 S^{\prime} \pi^{\prime}+S^{\prime \prime} \pi\right)+S \pi\left(U_{1}^{\prime \prime \prime}+\alpha^{2} U_{1}^{\prime}\right), \\
& i \alpha \operatorname{Re}\left[\left(\psi_{2}^{\prime \prime}-\alpha^{2} \psi_{2}\right)\left(U_{2}-c\right)-\psi_{2} U_{2}^{\prime \prime}\right]=\psi_{2}^{\prime \prime \prime \prime}-2 \alpha^{2} \psi_{2}^{\prime \prime}+\alpha^{4} \psi_{2}, \\
& \phi_{1}^{\prime \prime}-\alpha^{2} \phi_{1}=0, \\
& \phi_{2}^{\prime \prime}-\alpha^{2} \phi_{2}=0 .
\end{aligned}
$$

In the $(n, B n) \rightarrow(1,0)$ limit, these equations reduce to the linear stability equations used for Newtonian two-layer system under the influence of the electric field [39]. In the absence of an electric field, we recover the stability equations given in Refs. [40,41]. The classical Orr-Sommerfeld equation [42] can be obtained by setting $(n, B n) \rightarrow(1,0)$ and in the absence of electric field.
The eigenvalue $(c)$ and the eigenfunctions $\left(\psi_{1}, \psi_{2}, \phi_{1}\right.$ and $\left.\phi_{2}\right)$ are obtained via the solution of Eqs. (30)-(33) subject to the following boundary conditions for the disturbance.

$$
\begin{aligned}
& \psi_{1}=\psi_{1}^{\prime}=\phi_{1}=0, \text { at } y=0, \\
& \psi_{2}=\psi_{2}^{\prime}=\phi_{2}=0, \text { at } y=1, \\
& \psi_{1}=\psi_{2}, \\
& \psi_{1}^{\prime}-\psi_{2}^{\prime}+h\left(U_{1}^{\prime}-U_{2}^{\prime}\right)=0, \\
& \phi_{1}-\phi_{2}+h\left(\phi_{1}^{0^{\prime}}-\phi_{2}^{0^{\prime}}\right)=0, \\
& i \alpha \operatorname{Re}\left[\left\{\left(c-U_{1}\right) \psi_{1}^{\prime}+\psi_{1} U_{1}^{\prime}\right\}-\left\{\left(c-U_{2}\right) \psi_{2}^{\prime}+\psi_{2} U_{2}^{\prime}\right\}\right]-2 \mu_{1}^{0} \alpha^{2} \psi_{1}^{\prime}+3 \alpha^{2} \psi_{2}^{\prime}+ \\
& \quad \mu_{1}^{0}\left(\psi_{1}^{\prime \prime \prime}-\alpha^{2} \psi_{1}^{\prime}\right)+\mu_{1}^{0^{\prime}}\left(\psi_{1}^{\prime \prime}+\alpha^{2} \psi_{1}\right)+S^{\prime} U_{1}^{\prime} \pi+S U_{1}^{\prime} \pi^{\prime}+S U_{1}^{\prime \prime} \pi-\psi_{2}^{\prime \prime \prime}+ \\
& \quad i \alpha E_{0}\left(\phi_{2}^{\prime} \phi_{2}^{0^{\prime}}-\varepsilon_{r} \phi_{1}^{\prime} \phi_{1}^{0^{\prime}}\right)=-i \alpha^{3} \Gamma \frac{\left(\psi_{2}^{\prime}-\psi_{1}^{\prime}\right)}{\left(U_{2}^{\prime}-U_{1}^{\prime}\right)}, \\
& \psi_{2}^{\prime \prime}+\alpha^{2} \psi_{2}-\mu_{1}^{0}\left(\psi_{1}^{\prime \prime}+\alpha^{2} \psi_{1}\right)-S U_{1}^{\prime} \pi= \\
& \quad i \alpha E_{0}\left[\left(\phi_{2}-\frac{\psi_{2}}{\left(U_{2}-c\right)} \phi_{2}^{0^{\prime}}\right) \phi_{2}^{0^{\prime}}-\varepsilon_{r}\left(\phi_{1}-\frac{\psi_{1}}{\left(U_{1}-c\right)} \phi_{1}^{0^{\prime}}\right) \phi_{1}^{0^{\prime}}\right] .
\end{aligned}
$$

The kinematic condition for the disturbance is given by

$h=\left.\frac{\psi_{1}}{\left(c-U_{1}\right)}\right|_{h^{0}}=\left.\frac{\psi_{2}}{\left(c-U_{2}\right)}\right|_{h^{0}}$,

and the conservation charge density at the interface is given by

$i \alpha\left(U_{1}-c\right)\left(\varepsilon_{r} \phi_{1}^{\prime}-\phi_{2}^{\prime}\right)+i \alpha q^{0} \psi_{1}^{\prime}=\Lambda\left(\phi_{2}^{\prime}-\sigma_{r} \phi_{1}^{\prime}\right)$.

The linear perturbation equations (Eqs. (30)-(33)) along with the perturbed boundary conditions (Eqs. (34)-(42)) constitute an eigenvalue problem, which is given by

$\left[\begin{array}{cccc}\mathcal{A}_{11} & 0 & 0 & 0 \\ 0 & \mathcal{A}_{22} & 0 & 0 \\ 0 & 0 & \mathcal{A}_{33} & 0 \\ 0 & 0 & 0 & \mathcal{A}_{44}\end{array}\right]\left[\begin{array}{l}\psi_{1} \\ \psi_{2} \\ \phi_{1} \\ \phi_{2}\end{array}\right]=c\left[\begin{array}{cccc}\mathcal{B}_{11} & 0 & 0 & 0 \\ 0 & \mathcal{B}_{22} & 0 & 0 \\ 0 & 0 & 0 & 0 \\ 0 & 0 & 0 & 0\end{array}\right]\left[\begin{array}{l}\psi_{1} \\ \psi_{2} \\ \phi_{1} \\ \phi_{2}\end{array}\right]$,

where,

$$
\begin{aligned}
\mathcal{A}_{11}= & \alpha U_{1}\left(D^{2}-\alpha^{2}\right)-\alpha U_{1}^{\prime \prime}+\frac{i}{R e}\left[\mu_{1}^{0}\left(D^{4}-2 \alpha^{2} D^{2}+\alpha^{4}\right)\right. \\
+ & 2 \mu_{1}^{0^{\prime}}\left(D^{3}-\alpha^{2} D\right)+\mu_{1}^{0^{\prime \prime}}\left(D^{2}+\alpha^{2}\right)+\alpha^{2} S U_{1}^{\prime} \pi+ \\
& \left.S^{\prime \prime} U_{1}^{\prime} \pi+2 S^{\prime} U_{1}^{\prime} \pi^{\prime}+2 S^{\prime} U_{1}^{\prime \prime} \pi+S U_{1}^{\prime} \pi^{\prime \prime}+2 S U_{1}^{\prime \prime} \pi^{\prime}+S U_{1}^{\prime \prime \prime} \pi\right], \\
\mathcal{A}_{22}= & \alpha U_{2}\left(D^{2}-\alpha^{2}\right)-\alpha U_{2}^{\prime \prime}+\frac{i}{R e}\left(D^{4}-2 \alpha^{2} D^{2}+\alpha^{4}\right), \\
\mathcal{A}_{33}= & \left(D^{2}-\alpha^{2}\right), \mathcal{A}_{44}=\left(D^{2}-\alpha^{2}\right), \\
\mathcal{B}_{11}= & \alpha\left(D^{2}-\alpha^{2}\right), \quad B_{22}=\alpha\left(D^{2}-\alpha^{2}\right) .
\end{aligned}
$$

Here, $D \equiv d / d y$, and $\left(\psi_{1}, \phi_{1}\right)$ and $\left(\psi_{2}, \phi_{2}\right)$ are the eigenfunctions corresponding to the eigenvalue $c$ in the intervals $\left[0, h^{0}\right]$ and $\left[h^{0}, 1\right]$, respectively. This eigenvalue problem is solved by using the Chebyshev collocation method and a public domain software, LAPACK. This procedure allows the numerical calculation of the dispersion relations 


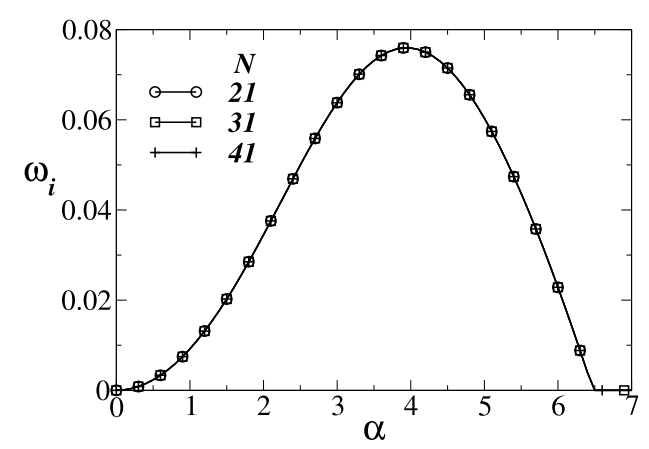

Fig. 3. The effect of increasing the order of Chebyshev polynomials, $N$, on the variation of $\omega_{i}$ with $\alpha$ (dispersion curve) obtained using a typical set of parameters, i.e $R e=10, m=10, n=1, \Gamma=1, h^{0}=0.3, \epsilon_{r}=2, \sigma_{r}=10, E_{0}=1$ and $B n=5$.

$\omega_{i}=\omega_{i}\left(\alpha ; R e, B n, n, m, \Gamma, E_{0}, \epsilon_{r}, \sigma_{r}, h^{0}\right)$. A similar technique has been used in Refs. [14,15]. We assume that $\Lambda$ is extremely large, i.e. $\Lambda \rightarrow \infty$, which implies that the charge relaxation time is very quick.

The stability code used in the present study was used in our earlier publications without electric field [14,15], wherein the code was extensively validated by comparing with the previously reported stability results associated with Newtonian fluids. Thus, here we only present the dependence of our numerical solutions upon mesh refinement in Fig. 3, which depicts the growth rate of the most unstable disturbance, $\omega_{i}$ against $\alpha$ (dispersion curve) for a typical set of parameters ( $R e=10$, $m=10, n=1, \Gamma=1, h^{0}=0.3, \epsilon_{r}=2, \sigma_{r}=10, E_{0}=1$ and $B n=5$ ). It is seen that the curves for different values of the order of Chebyshev polynomials, $N$, are virtually indistinguishable for $N \geq 21$. However, we generated the rest of the results presented in this study using $N=41$ to be in a safer side.

\section{Results and discussion}

We begin the presentation of our results by studying the effect of electric field on the linear stability behaviour of a pressure-driven two-layer channel flow consisting of two immiscible fluids, namely a Newtonian fluid (top layer) and a Herschel-Bulkley fluid (bottom layer) with $B n=5$ and $n=1$. In Figs. 4(a) and (b), we examine the dispersion curves for different values of the electrical Weber number, $E_{0}$, which characterises the strength of the electrical field for $h^{0}=0.3$ and 0.5, respectively. The rest of the parameters considered are $R e=10, m=10$, $\Gamma=1, \epsilon_{r}=10$ and $\sigma_{r}=2$ (hereafter, termed as 'base parameters'). It can be seen that the dispersion curves are paraboloidal with $\omega_{i}>$ 0 over a finite band of wavenumbers, indicating the presence of a linear instability. These curves exhibit well-defined "most-dangerous" and "cut-off" modes corresponding to the values of $\alpha$ for which $\omega_{i}$ is maximal (designated by " $A-D$ " in Fig. 4a and " $E-H$ " in Fig. 4(b) and beyond which $\omega_{i} \leq 0$, respectively. It can be observed in Figs. 4(a) and (b) that increasing $E_{0}$ increases the growth rate for both the values of $h^{0}$ considered. Close inspection also reveals that while the wavenumber of the most dangerous mode decreases as the value of $E_{0}$ increases for $h^{0}=0.3$, the opposite trend is apparent for $h^{0}=0.5$. Also the maximum growth rate at a particular value of $E_{0}$ for $h^{0}=0.3$ is higher than that for $h^{0}=0.5$. It indicates that decreasing the thickness of the non-Newtonian layer enhances the interfacial instability.

Using the formulation used in the present study, the conditions derived by Ozen et al. [28] for the stabilising influence of the applied electric field are

$\sigma_{r}^{2}<\epsilon_{r}$ for $\sigma_{r}<1$, and $\sigma_{r}^{2}>\epsilon_{r}$ for $\sigma_{r}>1$.

Similarly, the conditions for the destabilising influence of the applied electric field are

$\sigma_{r}^{2}>\epsilon_{r}$ for $\sigma_{r}<1$, and $\sigma_{r}^{2}<\epsilon_{r}$ for $\sigma_{r}>1$.
Note that in Figs. 4(a,b), $\sigma_{r}^{2}<\epsilon_{r}$ and $\sigma_{r}>1$. In Fig. 4(c), we investigate the effect of $E_{0}$ when $\sigma_{r}^{2}>\epsilon_{r}$ and $\sigma_{r}>1$. It can be seen in Fig. 4c that in this case, $E_{0}$ has a stabilising influence. Thus, it can be inferred that the behaviour of the electric field observed in the present study is consistent with that reported by Ozen et al. [28].

In order to understand the mechanism behind the instability associated with the electric field, we conduct an energy budget analysis similar to the one given in Ref. [4], which was performed for Newtonian fluids without any electric field. In the present study, the electric effect is included via the normal and tangential stresses. The energy budget equation is derived by taking the inner product of the horizontal and vertical components of the velocity disturbance equations with the respective velocity components. The resultant equation is then averaged over the wavelength of the disturbance, $\lambda=2 \pi / \alpha$ and integrated over the height of the channel to obtain the following energy budget equation:

$$
\sum_{i=1}^{2} K I N_{i}=\sum_{i=1}^{2} D I S_{i}+\sum_{i=1}^{2} R E Y_{i}+I N T
$$

where, $K I N_{i}$ is the temporal rate of change in disturbance kinetic energy, so $K I N>0$ implies instability, $D I S_{i}$ denotes the rate of change in the viscous dissipation of energy, $R E Y_{i}$ represents the "Reynolds stress" term, which determines the rate of transfer of energy from the basic flow to the disturbance and $I N T$ is associated with the existence of an interface. The term INT can be decomposed into TEN and TAN, which represent the work done by the velocity and stress disturbances in the directions normal and tangential to the interface, respectively, and are given by

$$
\begin{aligned}
& \operatorname{KIN}_{i}=\frac{1}{\lambda} \frac{d}{d t} \int_{a_{i}}^{b_{i}} d y \int_{0}^{\lambda} d x\left[\frac{1}{2}\left(\hat{u}_{i}^{2}+\hat{v}_{i}^{2}\right)\right], \\
& D I S_{i}=-\frac{1}{\lambda \operatorname{Re}} \int_{a_{i}}^{b_{i}} d y \int_{0}^{\lambda} \mu_{i} d x\left[2\left(\hat{u}_{i, x}\right)^{2}+\left(\hat{u}_{i, y}+\hat{v}_{i, x}\right)^{2}+2\left(\hat{v}_{i, y}\right)^{2}\right],
\end{aligned}
$$

$$
\begin{aligned}
& R E Y_{i}=\frac{1}{\lambda} \int_{a_{i}}^{b_{i}} d y \int_{0}^{\lambda} d x\left[-\hat{u}_{i} \hat{v}_{i} U_{i, y}\right] \\
& T E N=\frac{1}{\lambda \operatorname{Re}} \int_{0}^{\lambda}\left(\hat{v}_{1} \tau_{1, y y}-\hat{v}_{2} \tau_{2, y y}\right)_{y=h} d x \\
& \quad=\frac{1}{\lambda \operatorname{Re}} \int_{0}^{\lambda}\left(\hat{v}_{1} \Gamma \hat{h}_{x x}\right)_{y=h} d x+\frac{E_{0}}{\lambda \operatorname{Re}} \int_{0}^{\lambda}\left[\hat{v}_{1}\left(\epsilon_{r} \phi_{1}^{0^{\prime}} \hat{\phi}_{1}^{\prime}-\phi_{2}^{0^{\prime}} \hat{\phi}_{2}^{\prime}\right)\right]_{y=h} d x
\end{aligned}
$$

$$
\begin{aligned}
& T A N=\frac{1}{\lambda R e} \int_{0}^{\lambda}\left(\hat{u}_{1} \tau_{1, x y}-\hat{u}_{2} \tau_{2, x y}\right)_{y=h} d x+ \\
& \frac{E_{0}}{\lambda R e} \int_{0}^{\lambda}\left[\hat{u}_{2}\left(\hat{\phi}_{2, x}+\hat{h}_{x} \phi_{2}^{0^{\prime}}\right) \phi_{2}^{0^{\prime}}+\hat{u}_{1}\left(\hat{\phi}_{1, x}+\hat{h}_{x} \phi_{1}^{0^{\prime}}\right) \phi_{1}^{0^{\prime}}\right]_{y=h} d x,
\end{aligned}
$$

where subscript $i=1$ and 2 represent the non-Newtonian and Newtonian layers, respectively, such that for $i=1: \mu=\mu_{1}, a_{1}=0$ and $b_{1}=h^{0}$ and for $i=2: \mu=1, a_{2}=h^{0}$ and $b_{2}=1$. The components of the stress tensor are defined as

$\tau_{i, x y}=\mu_{i}\left(\hat{u}_{i, y}+\hat{v}_{i, x}\right)$ and $\tau_{i, y y}=-p_{i}+2 \mu_{i} \hat{v}_{i, y}$,

where $p_{i}$ denote the pressure disturbances.

The decomposition of rate of change of energies scaled by the total spatially averaged rate of change of disturbance kinetic energy, $K I N(=$ $K E N_{1}+K E N_{2}$ ) associated with points $A, B, C$ and $D$ in Fig. 4(a) which corresponds to $\alpha=4.4,3.8,3.78$ and 3.76 for $h^{0}=0.3$ is given in Table 2 . It can be observed that the work done by the velocity and stress disturbances in the direction tangential to the interface (TAN) provides the biggest positive contribution to the instability. This finding is similar to the one reported by other researchers for two-layer channel flow without electric field $[4,15,16]$. While the other contributions for point $A$ (without electric field) are negative, the "Reynolds stress" term associated with the Newtonian fluid $\left(R E Y_{2}\right)$ also makes positive contributions to the instability for points " $B-D$ " (with electric field) 
(a)

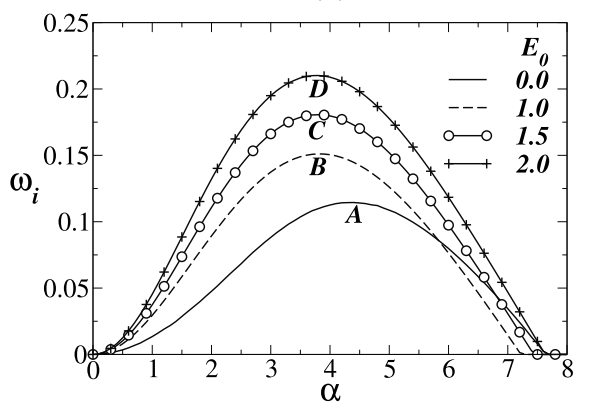

(b)

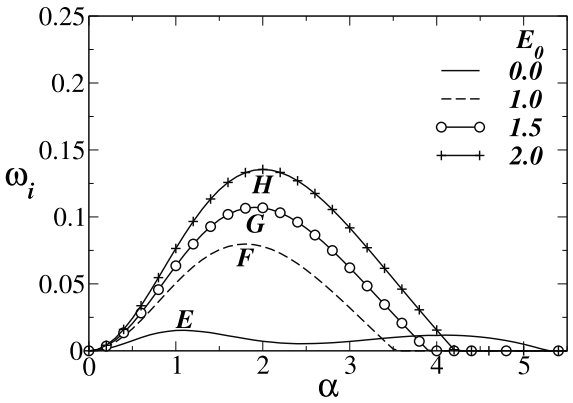

(c)

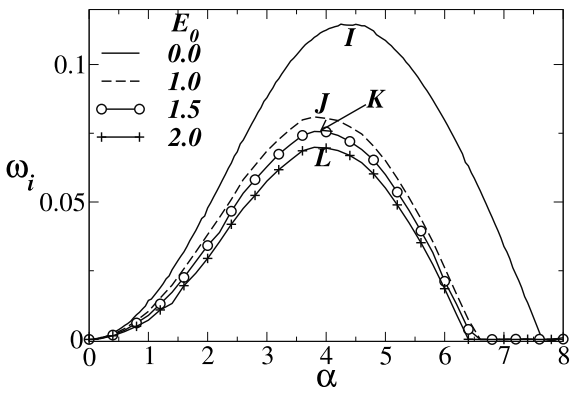

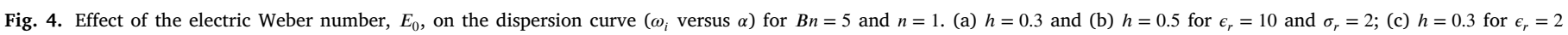
and $\sigma_{r}=5$. The rest of the parameters considered are $R e=10, m=10$ and $\Gamma=1$.

Table 2

Energy "budgets" for the points labelled $A, B, C$ and $D$ in Fig. 4(a).

\begin{tabular}{llllllll}
\hline Point & $\alpha$ & $R E Y_{1}$ & $R E Y_{2}$ & $D I S_{1}$ & $D I S_{2}$ & $T A N$ & $T E N$ \\
\hline$A$ & 4.4 & 0.0 & -0.0067 & -0.1317 & -0.8683 & 1.1193 & -0.0009 \\
$B$ & 3.8 & 0.0 & 0.0051 & -0.5286 & -0.4714 & 1.1583 & -0.0212 \\
$C$ & 3.78 & 0.0 & 0.0071 & -0.5359 & -0.4641 & 1.1502 & -0.0250 \\
$D$ & 3.76 & 0.0 & 0.0089 & -0.5441 & -0.4559 & 1.1993 & -0.0281 \\
\hline
\end{tabular}

Table 3

Energy "budgets" for the points labelled $E, F, G$ and $H$ in Fig. 4(b).

\begin{tabular}{llllllll}
\hline Point & $\alpha$ & $R E Y_{1}$ & $R E Y_{2}$ & $D I S_{1}$ & $D I S_{2}$ & $T A N$ & $T E N$ \\
\hline$E$ & 1.05 & 0.0 & 0.0017 & -0.4348 & -0.5652 & 1.1412 & -0.0001 \\
$F$ & 1.8 & 0.0001 & 0.0060 & -0.6968 & -0.3032 & 1.2617 & -0.0030 \\
$G$ & 1.9 & 0.0001 & 0.0076 & -0.7055 & -0.2945 & 1.2615 & -0.0042 \\
$H$ & 2.0 & 0.0001 & 0.0091 & -0.7146 & -0.2854 & 1.2584 & -0.0055 \\
\hline
\end{tabular}

Table 4

Energy "budgets" for the points labelled $I, J, K$ and $L$ in Fig. 4(c).

\begin{tabular}{llllllll}
\hline Point & $\alpha$ & $R E Y_{1}$ & $R E Y_{2}$ & $D I S_{1}$ & $D I S_{2}$ & $T A N$ & $T E N$ \\
\hline$I$ & 4.4 & 0.0 & -0.0067 & -0.1317 & -0.8683 & 1.1193 & -0.0009 \\
$J$ & 3.8 & 0.0 & -0.0091 & -0.2401 & -0.2273 & 0.5726 & -0.0054 \\
$K$ & 3.8 & 0.0 & -0.0110 & -0.4021 & -0.3819 & 0.8926 & -0.0085 \\
$L$ & 3.8 & 0.0 & -0.0115 & -0.4481 & -0.2363 & 0.7894 & -0.0049 \\
\hline
\end{tabular}

in Fig. 4(a). Close inspection of the $R E Y_{2}$ values in Table 2 reveals that increasing the value of the electrical Weber number, $E_{0}$ increases the positive contribution of this term towards the instability.

The energy budgets for points $E, F, G$ and $H$ corresponds to $\alpha=$ $1.05,1.8,1.9$ and 2 in Fig. $4 \mathrm{~b}$ for $h^{0}=0.5$ are presented in Table 3. For $h^{0}=0.5$, it can be seen that $T A N, R E Y_{1}$ and $R E Y_{2}$ provide positive contributions to the instability. In this case, albeit small, $R E Y_{1}$ also makes a positive contribution towards the instability. It can be observed that increasing the electric field strength (increasing $E_{0}$ ) increases the value of $R E Y_{2}$. The rest of the terms are negative indicating that they have stabilising contributions to the instability observed. In Table 4, the energy "budgets" for the points labelled $I, J, K$ and $L$ in Fig. 4(c), have been presented. It can be observed that $T A N$ remains the biggest positive contributor to the growth of the disturbance. However, unlike Table 2 for $\epsilon_{r}=10$ and $\sigma_{r}=2$, the negative contribution of $R E Y_{2}$ increases with increasing the strength of the applied electric field for $\epsilon_{r}=2$ and $\sigma_{r}=5$, thereby stabilising the flow. Thus, it can be concluded that the work done by the velocity and stress disturbances in the direction tangential to the interface $(T A N)$ and the rate of transfer of energy from the basic flow to the disturbance in the Newtonian layer $\left(R E Y_{2}\right)$ are the main source of instability observed in our study. The profiles of the real and imaginary parts of the eigenfunction ( $\psi_{1}$ and $\psi_{2}$ associated with the bottom and top layers) are shown in Figs. 5(a,b) and (c,d) for points $A, B, C$ and $D$ in Fig. 4(a) and points $E, F, G$ and $H$ in Fig. $4 \mathrm{~b}$, respectively. It can be seen that the streamwise disturbance velocity (gradient of $\psi_{1}$ and $\psi_{2}$ ) undergoes a discontinuity at the interface, $h^{0}=0.3$ and 0.5 in Figs. 5(a,b) and (c,d), respectively. This also suggests that the observed instability is due to the presence of the sharp interface and increasing the strength of the electric field has a significant effect on the eigenfunction which leads to an increase in instability.

We also investigate the effect of the electrical properties on the interfacial stability, by varying the electrical permittivity ratio $\left(\epsilon_{r}\right)$ for $\sigma_{r}=5.0$ and conductivity ratio for $\epsilon_{r}=2.0$ in Fig. 6(a) and (b), respectively. The other parameters are fixed as $h^{0}=0.5, B n=5$, $E_{0}=1, R e=10, m=10$ and $\Gamma=1$. It can be seen that increasing the electrical permittivity ratio and decreasing the electrical conductivity ratio destabilises the flow for the parameters considered in the present study. A similar behaviour is also observed by Ozen et al. [28] in the case of Newtonian fluid layers.

Next, we conduct a parametric study by varying the properties of the Herschel-Bulkley fluid, namely the Bingham number $(B n)$ and the flow index $(n)$. In Figs. $7(\mathrm{a}, \mathrm{c})$ and $(\mathrm{b}, \mathrm{d})$, we investigate the effects of varying the Bingham number, $B n$ on the linear stability characteristic for $E_{0}=0$ (without electric field) and $E_{0}=2.0$, respectively. The panels (a,b) and (c,d) are for $h^{0}=0.3$ and $h^{0}=0.5$, respectively. The rest of the parameters are the same as the "base parameters". In Fig. 7(a) for $h^{0}=0.3$ and without any applied electric field, increasing the value of $B n$ can be seen as resulting in non-monotonic dependency on the maximum growth rate. The maximum growth rate, $\omega_{i, \max }$ increases up to $B n=5$ and then decreases; it can be clearly seen that $\omega_{i, \max }$ for 
(a)

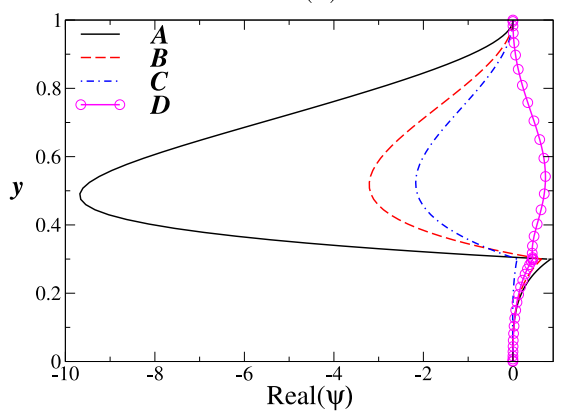

(c)

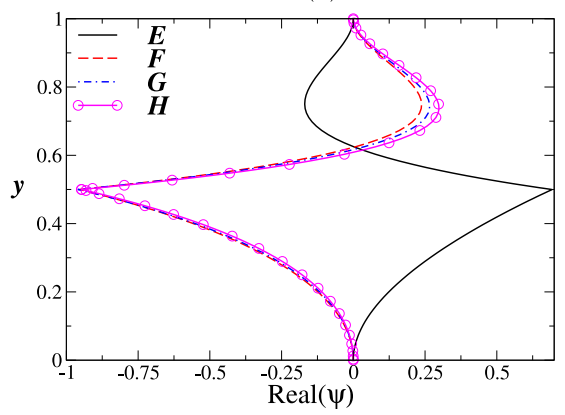

(b)

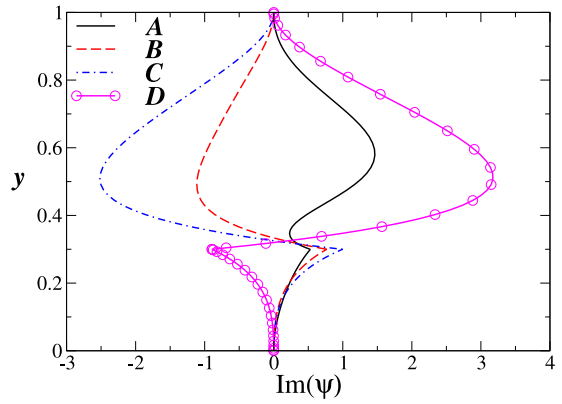

(d)

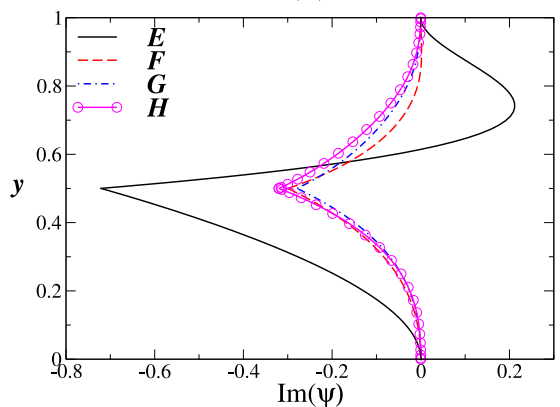

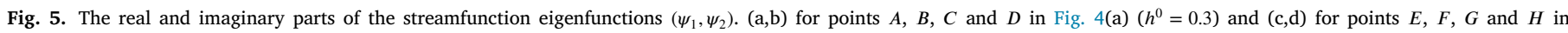
Fig. 4(b) $\left(h^{0}=0.5\right)$

(a)

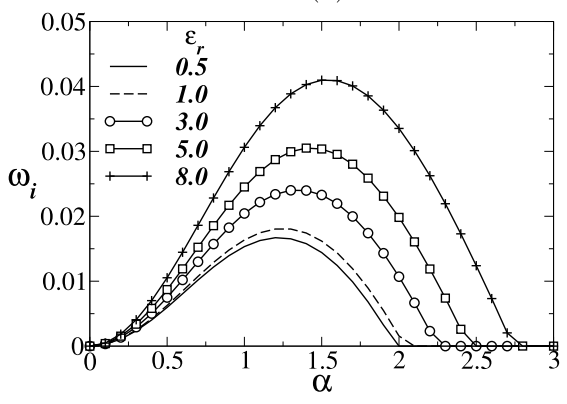

(b)

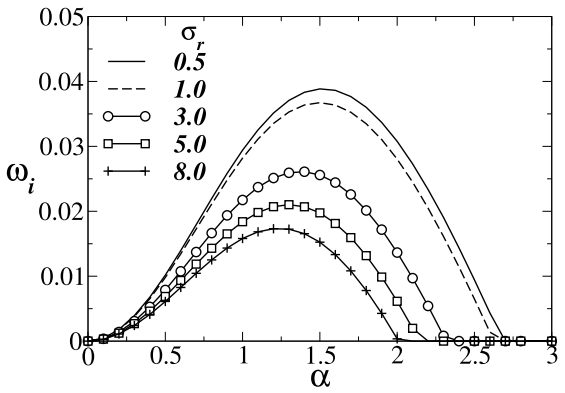

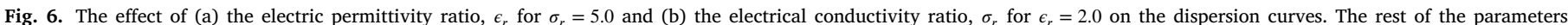
used are $h^{0}=0.5, B n=5, E_{0}=1, R e=10, m=10$ and $\Gamma=1$.

$B n=8$ is lower than that of $B n=5$ in Fig. 7(a). This reduction in growth rate for $B n=8$ can be due to the fact that for $B n=8$, the gradient of the basic state velocity profile in the non-Newtonian layer approaches zero. A similar behaviour was observed in Ref. [43]. For $h^{0}=0.5$ and without any applied electric field (Fig. 7(b)), it can be observed that the growth rate at $\alpha \approx 1.1$ is constant for all values of $B n$, but another mode with higher growth rate than the unstable mode at $\alpha \approx 1.1$ appears in the high wavenumber region. In contrast, in the case with electric field Figs. 7(b,d), increasing the value of $B n$ decreases the maximum growth rate, $\omega_{i, \max }$ for both values of $h^{0}$ considered. Comparison of the dispersion curves in Figs. 7(a,c) and Figs. 7(b,d) also reveals that the external electric field destabilises the system for all $B n$ values. The energy budget analysis presented in Tables 2 and 3 reveals that increasing the strength of the electric field (electrical Weber number) increases the positive contribution of the "Reynolds stress" term of the Newtonian layer $\left(R E Y_{2}\right)$ and thereby increasing the growth rate of the disturbances for each value of $B n$. This surpasses the stabilising influence from other disturbance energy terms, which in turn suppresses the non-monotonic behaviour observed as we increase the value of $B n$.
The variations of the growth rate of the most dangerous mode, $\omega_{i, \max }$ with $B n$ for different values of $E_{0}$ are plotted in Figs. 8a and b for $h^{0}=0.3$ and $h^{0}=0.5$, respectively. The rest of the parameters are the same as those used to generate Fig. 7. The non-monotonic dependency on the maximum growth rate with $B n$ discussed in Fig. 7(a) for no electric field $\left(E_{0}=0\right)$ case can be clearly visible in Fig. 8a. This non-monotonic behaviour is also evident up to $E_{0}=1$. However, for $E_{0} \geq 1.5$, it can be seen that increasing $B n$ gradually decreases the value of $\omega_{i, \max }$ for $h^{0}=0.3$ (see Fig. 8a). In the case of a thicker nonNewtonian layer $\left(h^{0}=0.5\right)$, increasing $B n$ has a minimal effect on the $\omega_{i, \max }$ for each value of $E_{0}$.

In Fig. 9, the effect of the flow index, $n$ is investigated in the absence and presence of the electric field with the electrical Weber number, $E_{0}=2$ for $h^{0}=0.5$ and $B n=2$. In Fig. 9a with $E_{0}=0$, it can be seen that $n<1$ (shear-thinning) and $n>1$ (shear-thickening) fluids exhibit higher growth of the most unstable mode. In the shear thickening case $(n>1)$, increasing $n$ not only increases the value of $\omega_{i, \max }$ but also the range of unstable wave numbers (i.e. increases the value of the "cutoff" wave number). In the presence of the electric field $\left(E_{0}=2\right)$, it can be seen in Fig. 9b that the maximum growth rate of the disturbance exhibits a similar non-monotonic trend, but about $n=1.5$ instead of 
(a)

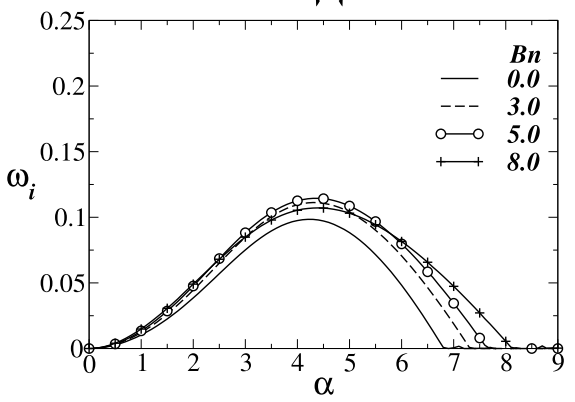

(c)

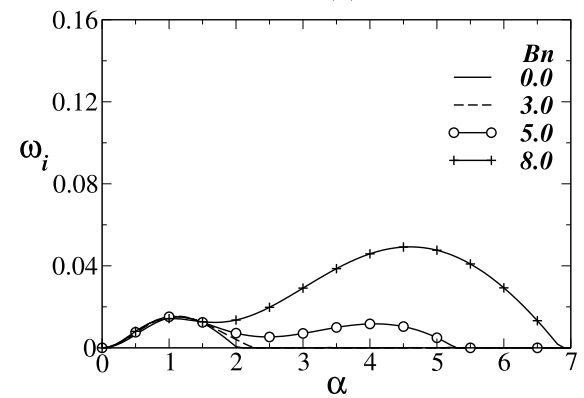

(b)

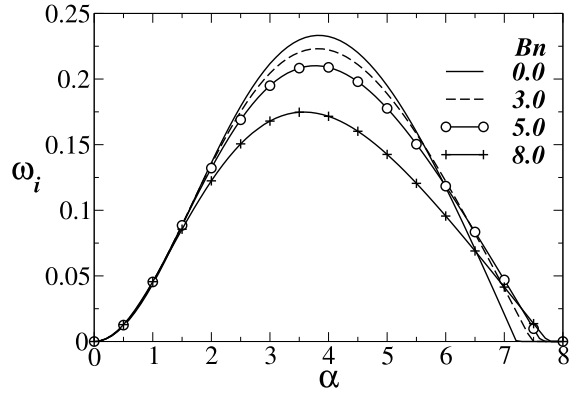

(d)

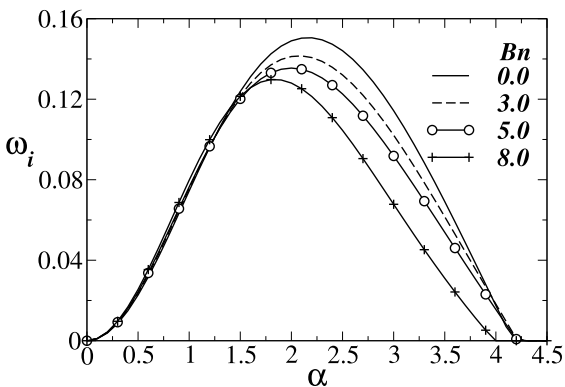

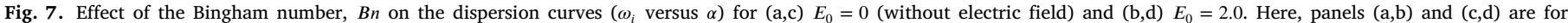
$h^{0}=0.3$ and $h^{0}=0.5$, respectively. Here, $n=1$ and the rest of the parameters are the same as the "base parameters".

(a)

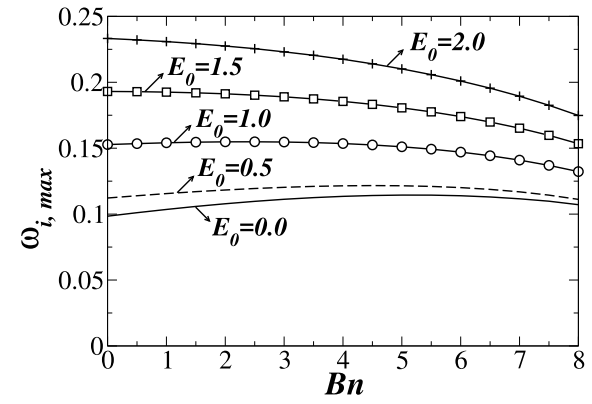

(b)

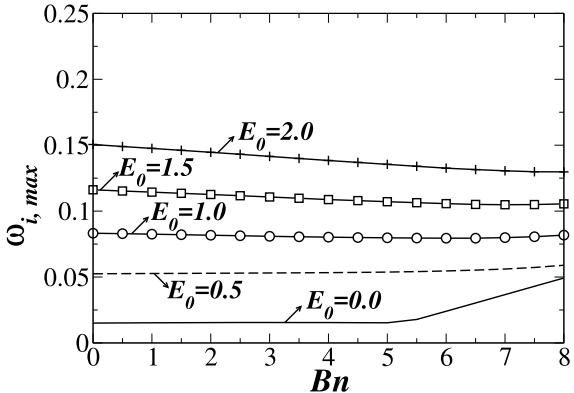

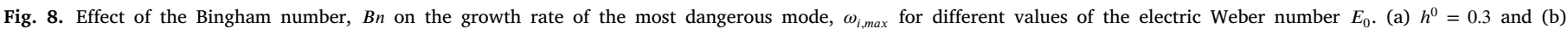
$h^{0}=0.5$. Here, $n=1$ and the rest of the parameters are the same as the "base parameters".

(a)

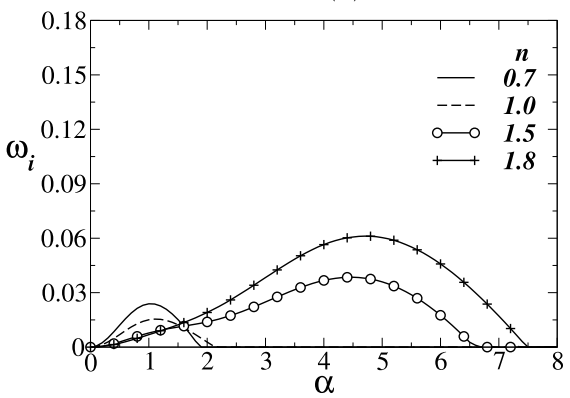

(b)

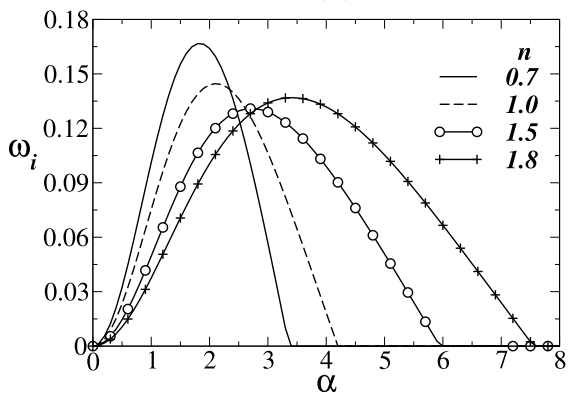

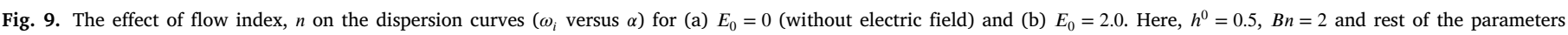
are the same as the "base parameters".

$n=1$ as shown in Fig. 9a. It is also observed in Fig. 9b that the range of unstable wave numbers increases with increasing the value of $n$. Comparison of the dispersion curves in Figs. 9a and Fig. 9b reveals that the electric field destabilises the flow for both the shear-thinning and shear-thickening fluids.
Then, we investigate the effect of the thickness of the bottom layer $\left(h^{0}\right)$ on the linear stability behaviour of the most-unstable mode. In Figs. 10a,b, we present the variations of $\omega_{i, \max }$ with $h^{0}$ for different values of $E_{0}$ for the Newtonian $(B n=0)$ and non-Newtonian $(B n=5)$ bottom layer. In both the cases, $n=1$ and the rest of the parameters are the same as the "base parameters". It can be seen that the Newtonian 
(a)

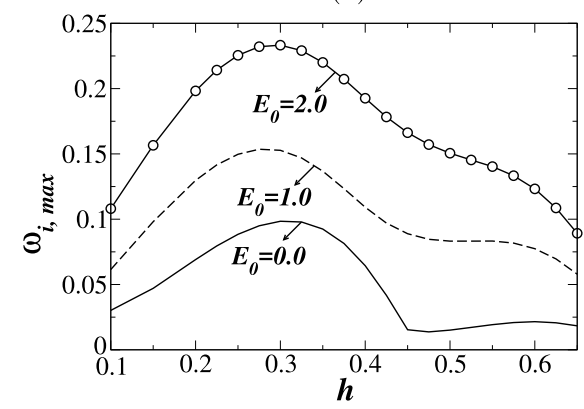

(b)

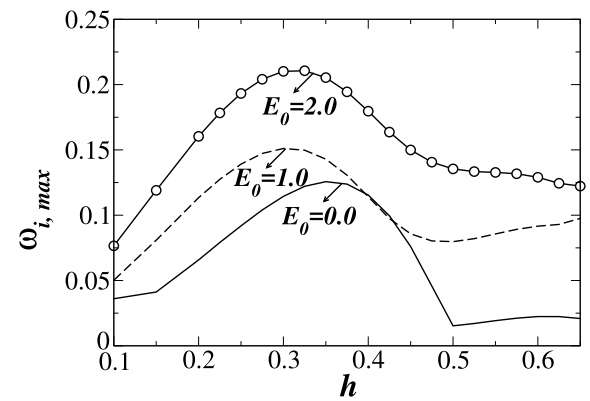

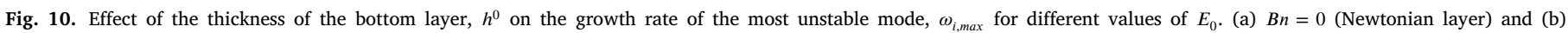
$B n=5$ (Bingham fluid layer). Here, $n=1$ and the rest of the parameters are the same as the "base parameters".

(a)

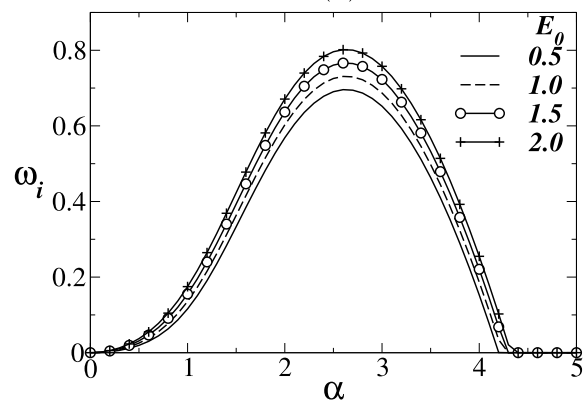

(b)

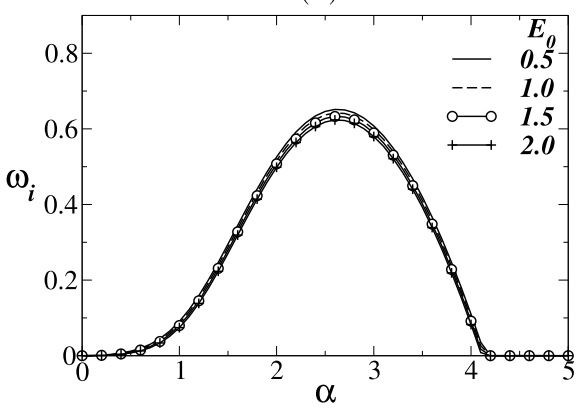

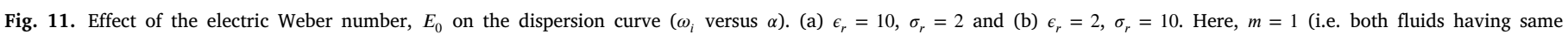
viscosity) and the rest of the parameters are for $B n=5, n=1, h=0.3, R e=10$ and $\Gamma=1$.

and the non-Newtonian cases show a similar trend, i.e. the maximum growth rate of the disturbance occurs for an intermediate value of $h^{0}$ and increases with increasing the value of $E_{0}$. A similar linear instability behaviour was also observed in Ref. [14] without electric field.

Finally, to check whether the destabilisation observed in the presence of the electric field is due to the viscosity contrast between the fluids, in Fig. 11(a) and (b), we study the effect of the electric Weber number, $E_{0}$ on the growth rate of the disturbance, when both the fluids have the same viscosity $(m=1)$ for $\epsilon_{r}=10, \sigma_{r}=2$ and $\epsilon_{r}=2, \sigma_{r}=10$, respectively. The rest of the parameters are for $B n=5, n=1, h=0.3$, $R e=10$ and $\Gamma=1$. It can be seen that increasing the value of $E_{0}$ has a destabilising and stabilising influence when $\sigma_{r}^{2}<\epsilon_{r}$ and $\sigma_{r}^{2}>\epsilon_{r}$, respectively for $\sigma_{r}>1$. Thus, it can be concluded that the influence of the electric Weber number is not due to the viscosity contrast, which is the main reason behind the interfacial instability without the external electric field.

\section{Concluding remarks}

The linear stability characteristics of a pressure-driven two-layer configuration of two immiscible fluids subjected to an electric field normal to the flow is investigated by conducting a linear stability analysis. The bottom layer is a non-Newtonian fluid characterised by the Herschel-Bulkley model, while the top layer is a Newtonian fluid. In order to isolate the effect of the electric field, we considered iso-dense fluids. The linear stability equations are derived and solved using an accurate spectral Chebyshev collocation method. The stability behaviour of the flow with a bottom non-Newtonian fluid layer is contrasted with the flow when both the layers are Newtonian fluids. The various parameters influencing the linear stability behaviour are the electric field strength, the electric permittivity and electrical conductivity ratios of the non-Newtonian to the Newtonian fluids, the Bingham number, the flow index and the thickness of the bottom layer. We ensured that the parameters considered does not lead to any unyielded region in the flow. We observe that increasing the electric permittivity ratio $\left(\epsilon_{r}\right)$ and decreasing the electrical conductivity ratio $\left(\sigma_{r}\right)$ keeping the rest of the parameters fixed enhances the growth rate of the disturbances. We also found that increasing the electric field strength destabilises and stabilises the flow when $\sigma_{r}^{2}<\epsilon_{r}$ and $\sigma_{r}^{2}>\epsilon_{r}$, respectively for $\sigma_{r}>1$. This finding is consistent with that reported by Ozen et al. [28] for Newtonian fluids. The "Reynolds stress" of the Newtonian layer and the work done by the velocity and stress disturbances tangential to the interface are found to be the mechanism of the instability due to the electric effect. The other findings obtained from the parametric study conducted are (i) increasing Bingham number can be stabilising or destabilising depending on the thickness of the non-Newtonian layer and the maximum disturbance growth occurs at an optimum value of non-Newtonian layer thicknesses, (ii) increasing the flow index from the shear-thinning $(n<1)$ to the shear-thickening $(n>1)$ has a nonmonotonic effect on the maximum growth rate of the disturbance, and (iii) the disturbance exhibits a maximum growth for an optimal value of the thickness of the non-Newtonian layer. The present study finds its applications in microfluidic and electronic cooling systems.

\section{Declaration of competing interest}

The authors declare that they have no known competing financial interests or personal relationships that could have appeared to influence the work reported in this paper.

\section{Acknowledgements}

K. C. S. thanks Science and Engineering Research Board, India for providing financial support through the grant number, MTR/2017/ 000029. G. K. would like to acknowledge the research fellowship provided by the Council of Scientific and Industrial Research, India through the grant number, 09/1001(0022)/2016-EMR-I. 


\section{References}

[1] A. Amiri, A. Eslami, R. Mollaabbasi, F. Larachi, S.M. Taghavi, Removal of a yield stress fluid by a heavier newtonian fluid in a vertical pipe, J. Non-Newton. Fluid Mech. 268 (2019) 81-100.

[2] A. Eslami, R. Mollaabbasi, A. Roustaei, S.M. Taghavi, Pressure-driven displacement flows of yield stress fluids: Viscosity ratio effects, Can. J. Chem. Eng. 97 (11) (2019) 2804-2817.

[3] C.S. Yih, Instability due to viscous stratification, J. Fluid Mech. 27 (1967) $337-352$.

[4] P.A.M. Boomkamp, R.H.M. Miesen, Classification of instabilities in parallel two-phase flow, Int. J. Multiph. Flow. 22 (1996) 67-88.

[5] S.G. Yiantsios, B.G. Higgins, Numerial solution of eigenvalue problems using the compound matrix-method, J. Comput. Phys. 74 (1988) 25.

[6] S.G. Yiantsios, B.G. Higgins, Linear stability of plane poiseuille flow of two superposed fluids, Phys. Fluids 31 (1988) 3225-3238.

[7] E.J. Hinch, A note on the mechanism of the instability at the interface between two shearing fluids, J. Fluid Mech. 144 (1984) 463-465.

[8] F. Charru, E.J. Hinch, 'Phase diagram' of interfacial instabilities in a two-layer couette flow and mechanism for the long-wave instability, J. Fluid Mech. 414 (2000) 195-223.

[9] M.J. South, A.P. Hooper, Linear growth in two-fluid plane Poiseuille flow, J. Fluid Mech. 381 (2001) 121-139.

[10] A. Pinarbasi, A. Liakopoulos, Stability of two-layer Poiseulle flow of CarreauYasuda and Bingham-like fluids, J. Non-Newt. Fluid Mech. 57 (1995) $227-241$.

[11] I.A. Frigaard, S.D. Howison, I.J. Sobey, On the stability of Poiseuille flow of a Bingham fluid, J. Fluid Mech. 263 (1994) 133-150.

[12] I.A. Frigaard, Super-stable parallel flows of multiple visco-plastic fluids, J. Non-Newt. Fluid Mech. 100 (2001) 49-76.

[13] I. Frigaard, C. Nouar, On three-dimensional linear stability of Poiseuille flow of Bingham fluids, Phys. Fluids 15 (10) (2003) 2843-2851.

[14] K.C. Sahu, P. Valluri, P.D.M. Spelt, O.K. Matar, Linear instability of pressuredriven channel flow of a Newtonian and Herschel-Bulkley fluid, Phys. Fluids 19 (2007) 122101.

[15] K.C. Sahu, O.K. Matar, Three-dimensional linear instability in pressure-driven two-layer channel flow of a Newtonian and a Herschel-Bulkley fluid, Phys. Fluids 22 (2010) 112103.

[16] R. Usha, K.C. Sahu, Interfacial instability in pressure-driven core-annular pipe flow of a newtonian and a Herschel-Bulkley fluid, J. Non-Newton. Fluid Mech. 271 (2019) 104144.

[17] R. Govindarajan, K.C. Sahu, Instabilities in viscosity-stratified flows, Ann. Rev. Fluid Mech. 46 (2014) 331-353.

[18] G.I. Taylor, A.D. McEwan, The stability of a horizontal fluid interface in a vertical electric field, J. Fluid Mech. 22 (1) (1965) 1-15.

[19] J.R. Melcher, C.V. Smith, Electrohydrodynamic charge relaxation and interfacial perpendicular-field instability, Phys. Fluids 12 (4) (1969) 778-790.

[20] D.A. Saville, Electrohydrodynamics: The Taylor-Melcher leaky dielectric model, Ann. Rev. Fluid Mech. 29 (1) (1997) 27-64.

[21] O. Ozen, N. Aubry, D.T. Papageorgiou, P.G. Petropoulos, Monodisperse drop formation in square microchannels, Phys. Rev. Lett. 96 (14) (2006) 144501.

[22] A.O. El Moctar, N. Aubry, J. Batton, Electro-hydrodynamic micro-fluidic mixer, Lab Chip 3 (4) (2003) 273-280.
[23] I. Glasgow, J. Batton, N. Aubry, Electroosmotic mixing in microchannels, Lab Chip 4 (6) (2004) 558-562.

[24] H. Lin, B.D. Storey, M.H. Oddy, C.H. Chen, J.G. Santiago, Instability of electrokinetic microchannel flows with conductivity gradients, Phys. Fluids 16 (6) (2004) 1922-1935.

[25] J.F. Hoburg, J.R. Melcher, Internal electrohydrodynamic instability and mixing of fluids with orthogonal field and conductivity gradients, J. Fluid Mech. 73 (2) (1976) 333-351.

[26] R.V. Craster, O.K. Matar, Electrically induced pattern formation in thin leaky dielectric films, Phys. Fluids 17 (3) (2005) 032104.

[27] R.M. Thaokar, V. Kumaran, Electrohydrodynamic instability of the interface between two fluids confined in a channel, Phys. Fluids 17 (8) (2005) 084104.

[28] O. Ozen, N. Aubry, D.T. Papageorgiou, P.G. Petropoulos, Electrohydrodynamic linear stability of two immiscible fluids in channel flow, Electrochim. Acta 51 (25) (2006) 5316-5323.

[29] S.Y. Chou, L. Zhuang, L. Guo, Lithographically induced self-construction of polymer microstructures for resistless patterning, Appl. Phys. Lett. 75 (7) (1999) 1004-1006.

[30] E. SchaÈffer, T. Thurn-Albrecht, T.P. Russell, U. Steiner, Electrically induced structure formation and pattern transfer, Nature 403 (6772) (2000) 874-877.

[31] L. Wu, S.Y. Chou, Electrohydrodynamic instability of a thin film of viscoelastic polymer underneath a lithographically manufactured mask, J. Non-Newton. Fluid Mech. 125 (2-3) (2005) 91-99.

[32] G. Tomar, V. Shankar, A. Sharma, G. Biswas, Electrohydrodynamic instability of a confined viscoelastic liquid film, J. Non-Newton. Fluid Mech. 143 (2-3) (2007) $120-130$.

[33] N.T.M. Eldabe, Electrohydrodynamic stability of two superposed elasticoviscous liquids in plane couette flow, J. Math. Phys. 28 (11) (1987) 2791-2800.

[34] N.T. Eldabe, Electrohydrodynamic stability of two stratified power law liquids in couette flow, Il Nuovo Cimento B (1971-1996) 101 (2) (1988) 221-235.

[35] G. Ersoy, A.K. Uguz, Electro-hydrodynamic instability in a microchannel between a newtonian and a non-Newtonian liquid, Fluid Dyn. Res. 44 (3) (2012) 031406.

[36] A. Nurocak, A.K. Uguz, Effect of the direction of the electric field on the interfacial instability between a passive fluid and a viscoelastic polymer, Eur. Phys. J-Spec. Top. 219 (1) (2013) 99-110.

[37] S.I. Kaykanat, A.K. Uguz, The linear stability between a Newtonian and a powerlaw fluid under a normal electric field, J. Non-Newton. Fluid Mech. 277 (2020) 104220.

[38] V.T.S.R. Kumar Reddy, V.M. Janardhanan, K.C. Sahu, Effects of wall-heating on the linear instability characteristics of pressure-driven two-layer channel flow, Chem. Eng. Sci. 66 (23) (2011) 6272-6279.

[39] F. Li, O. Ozen, N. Aubry, D.T. Papageorgiou, P.G. Petropoulos, Linear stability of a two-fluid interface for electrohydrodynamic mixing in a channel, J. Fluid Mech. 583 (25) (2007) 347-377.

[40] K.C. Sahu, Double-diffusive instability in core-annular pipe flow, J. Fluid Mech. 789 (2016) 830-855.

[41] K.C. Sahu, Linear instability in two-layer channel flow due to double-diffusive phenomenon, Phys. Fluids 32 (2) (2020) 024102.

[42] P.G. Drazin, W.H. Reid, Hydrodynamic Stability, Cambridge University Press, Cambridge, 1985.

[43] K.C. Sahu, Linear instability in a miscible core-annular flow of a Newtonian and a Bingham fluid, J. Non-Newton. Fluid Mech. 264 (2019) 159-169. 